\title{
A INTERDISCIPLINARIDADE COMO ESTRATÉGIA DE ESTÍMULO NO DESENVOLVIMENTO DE UMA FERRAMENTA COMPUTACIONAL DE APOIO AO ENSINO NA ÁREA DE ELETROTÉCNICA
}

\section{DOI: 10.37702/2175-957X.COBENGE.2021.3546}

Fabiola Graziela Noronha Barros - fabiola.graziela@ifpa.edu.br Instituto Federal do Pará Rua Madeira 32

68455-750 - Tucuruí - PA

Wellington Alex dos Santos Fonseca - wellington.fonseca@ifpa.edu.br Instituto Federal do Pará Rua Madeira 32 68455-750 - Tucuruí - PA

Alberth Davi do Carmo Silvino - davisilvino456@gmail.com Instituto Federal do Pará Rua Havaí 69 68257-210 - Tucuruí - PA

Eully Alves de Almeida - eully2002@gmail.com Instituto Federal do Pará Rodovia Transamazônica 330 68473-000 - Novo Repartimento - PA

Resumo: Este trabalho apresenta o resultado da interdisciplinaridade utilizada como estratégia de estímulo ao desenvolvimento de uma ferramenta computacional de apoio ao ensino na área de eletrotécnica. O cerne do trabalho encontra-se no conjunto de ações realizadas pelos discentes, do curso Técnico em Eletrotécnica do IFPA, durante o planejamento e execução das etapas que culminaram no desenvolvimento de um aplicativo para dispositivo móvel. 0 aplicativo, desenvolvido utilizando a ferramenta Kodular, automatizou o processo de determinação do valor da resistência e tolerância para resistores de 4 e 5 anéis.

Palavras-chave: : Interdisciplinaridade. Desenvolvimento de aplicativo. Circuitos 


\section{(C. COBENGE e IV Simpósio Internacional de Educação em Engenharia

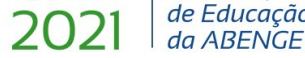 28 a 30 de SETEMBRO

elétricos. Eletrotécnica. 


\section{A INTERDISCIPLINARIDADE COMO ESTRATÉGIA DE ESTÍMULO NO DESENVOLVIMENTO DE UMA FERRAMENTA COMPUTACIONAL DE APOIO AO ENSINO NA ÁREA DE ELETROTÉCNICA}

\section{INTRODUÇÃO}

A resistência é a característica elétrica dos materiais, que representa a oposição à passagem da corrente elétrica (MARKUS, 2011). Para resistores de maiores potências, o valor nominal da resistência fixa e a tolerância podem ser gravados em sua estrutura. Para resistores de menores potências, utilizam-se anéis coloridos gravados em sua estrutura, que a partir de um código de cores preestabelecido, informam seus valores nominais e suas tolerâncias.

O valor nominal da resistência fixa e a tolerância de um resistor podem ser determinados por meio dos algarismos representados pelos anéis coloridos, de forma manual, ou determinados por meio de instrumentos de medidas, como o multímetro ou o ohmímetro, ou ainda por meio de um programa computacional desenvolvido para essa finalidade.

Neste sentido, este trabalho apresenta uma ferramenta computacional desenvolvida em Kodular, plataforma baseada no App Inventor, para automatizar, o processo de determinação do valor da resistência fixa e a tolerância de resistores que possuem 4 e 5 faixas de cores. O aplicativo foi desenvolvido por alunos do curso Técnico em Eletrotécnica, após cursarem as disciplinas de Algoritmos e Programação, e Circuitos Elétricos CC e CA. Desta forma, o objetivo geral deste trabalho foi incentivar e despertar o interesse vocacional dos discentes pela área da eletrotécnica/engenharia elétrica, por meio do desenvolvimento da iniciação científica júnior em conjunto com a interdisciplinaridade de duas ou mais disciplinas por eles cursadas, e nesse sentido, os objetivos foram alcançados.

\section{REFERENCIAL TEÓRICO}

Os resistores são componentes usados nos circuitos elétricos e eletrônicos para controlar a intensidade de corrente que passa pelos diversos componentes, bem com para controlar a tensão aplicada em cada parte do circuito. O funcionamento dos resistores está baseado na resistência elétrica que os materiais possuem de dificultarem a passagem da corrente elétrica. Quando um resistor é percorrido por uma corrente elétrica, ele tende a aquecer por conta do efeito Joule. A potência dissipada pelo resistor é igual ao produto da intensidade da corrente (MORA, et al., 2021).

Para determinar o valor de um resistor existem duas opções (PUCRS, 2001):

1. Por meio da utilização de instrumentos de medidas, como o Multímetro ou o Ohmímetro; ou

2. Por meio da leitura do próprio corpo do resistor através dos anéis de cores.

Convencionalmente, em sala de aula, utiliza-se a tabela a cores para se obter o valor da resistência elétrica, através de cálculos matemáticos manuais, entretanto, com o avanço tecnológico, a presença de sistemas programáveis estão cada vez mais presente no meio acadêmico e profissional, permitindo sua aplicação em tarefas antes realizadas manualmente. 
Ademais, a evolução de ferramentas de programação facilitou o desenvolvimento de aplicações computacionais, que permitem que o usuário possa criar aplicativos para smartphones, tablets e sites, sem a necessidade de ser expert em alguma linguagem de programação. Desta forma, o usuário pode criar propostas cada vez mais sofisticadas e que podem gerar produtos cada vez mais robustos e de qualidade.

Nesse sentido, a plataforma Kodular, evolução do Makeroid, criada pelo MIT (Massachusetts Institute of Technology), surge como uma ferramenta de ensino, no que tange a modelagem e desenvolvimento de ferramentas computacionais simples e aplicáveis a atividades relacionadas a área de circuitos elétricos.

Portanto, a união dos conhecimentos adquiridos pelos discentes nas disciplinas de algoritmos e programação e de circuitos elétricos permitiu o desenvolvimento de uma ferramenta computacional para dispositivos móveis que determina o valor da resistência e tolerância, baseado na sequência de cores no corpo do resistor informada ao programa, e que adicionalmente atende por comando de voz.

\section{METODOLOGIA}

O trabalho desenvolvido pode ser dividido em três etapas principais: (1) estudo, levantamento de informações e modelagem dos dados, (2) estudo da ferramenta Kodular e (3) desenvolvimento do aplicativo para dispositivo móvel.

Na primeira etapa, os discentes realizaram uma revisão sobre os principais conceitos de algoritmos e linguagem de programação, além dos assuntos relacionados à resistência elétrica e Leis de Ohm. Nesse momento, foi desenvolvido o algoritmo que implementa o funcionamento do programa. O algoritmo foi implementado e testado utilizando o VisuALG (Figura 1), que é um aplicativo para Windows que permite editar, interpretar e executar algoritmos com uma linguagem próxima ao português. A realização desta etapa permitiu a modelagem das informações que foram implementadas no programa em sua versão final.

Figura 1 - Trechos do algoritmo implementado em VisuALG.
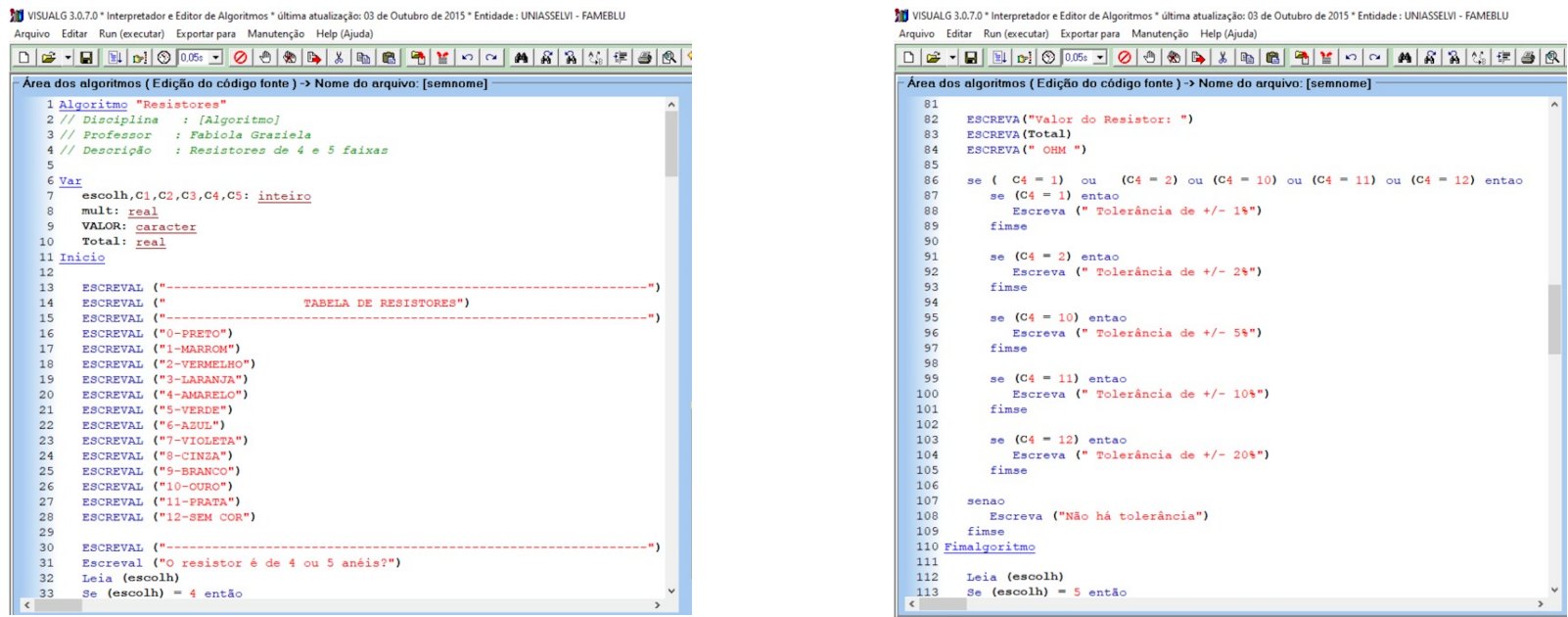

Fonte: Os autores.

A segunda etapa engloba o estudo da ferramenta de desenvolvimento de aplicativos Kodular. O Kodular é uma plataforma gratuita que oferece ferramentas e suporte para o usuário com o objetivo de facilitar o processo de criação de aplicativos. Nesse sentido, a 


\section{(C) COBENGE $2021 \mid \begin{aligned} & \text { e IV Simpósio Internacional } \\ & \text { de Educação em Engenharia } \\ & \text { da ABENGE }\end{aligned}$ 28 a 30 de SETEMBRO \\ Evento Online \\ "Formação em Engenharia: \\ Tecnologia, Inovação e Sustentabilidade"}

programação é realizada por meio de blocos lógicos (Figura 2), e conta com um layout simples e intuitivo, o que por sua vez, facilitou a construção do programa.

Figura 2 - Tela de desenvolvimento do aplicativo em Kodular.

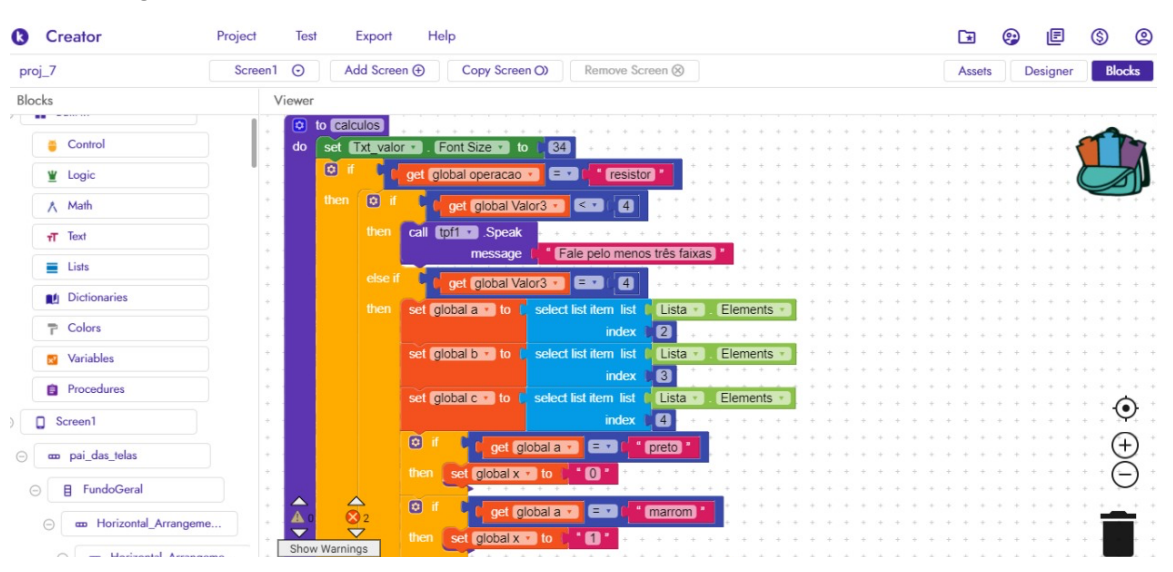

Fonte: Os autores.

$\mathrm{Na}$ terceira etapa, os discentes trabalharam no desenvolvimento do aplicativo. O resultado final, é um programa que determina o valor da resistência elétrica de resistores de 4 ou 5 anéis e suas respectivas tolerância, dada a sequência de cores dos anéis (Figura 3). A Figura 3 apresenta a tela inicial do programa onde pode-se observar o campo que permite digitas a sequência de cores do resistor. $O$ aplicativo também pode ser utilizado por comando de voz.

Figura 3 - Tela para a entrada da sequência de cores e tela de saída com o valor da resistência determinada.
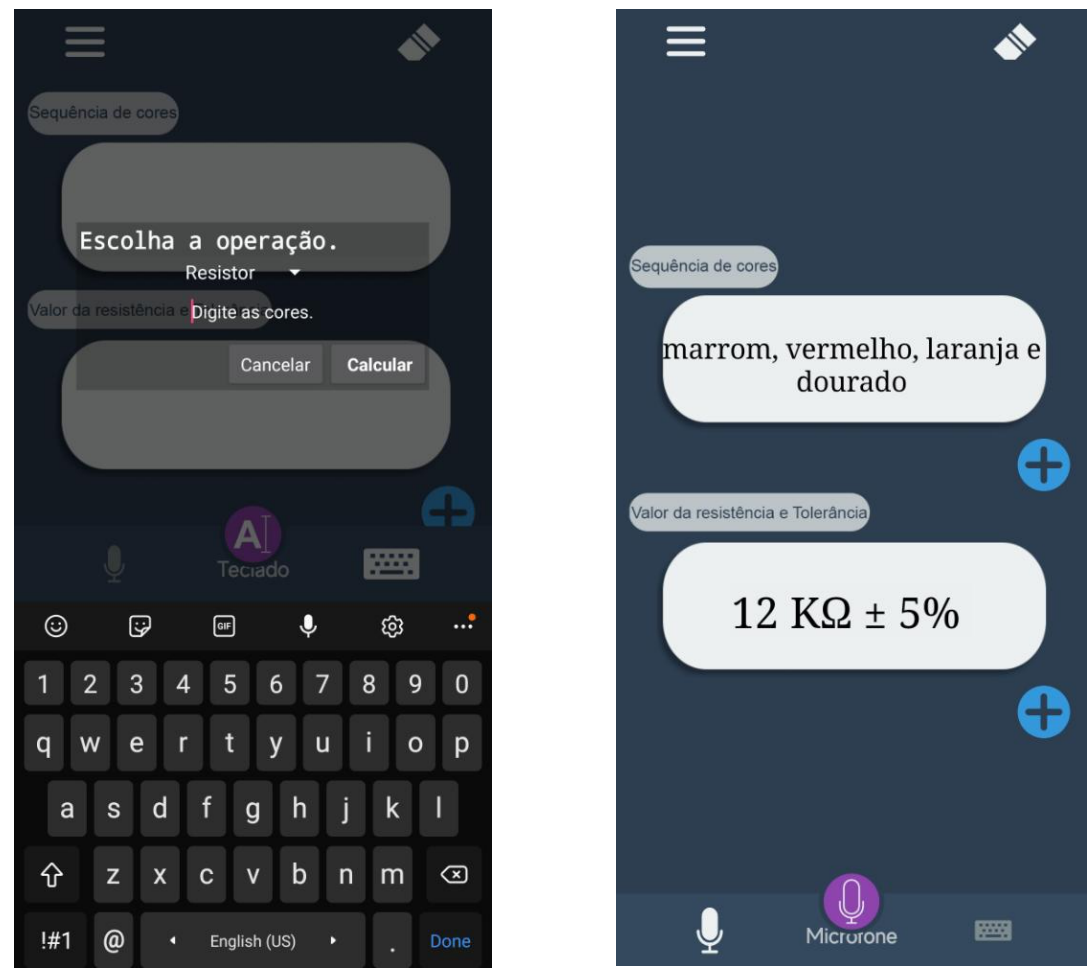

Fonte: Os autores. 


\subsection{Interdisciplinaridade como estratégia de motivação}

Um dos principais divisores de água no que se refere a continuidade de um estudante em uma determinada de área de conhecimento são as experiências iniciais destes com a área. No que tange as áreas das engenharias, a dificuldade inicial com as disciplinas de exatas durante o ensino fundamental e médio torna-se um fator determinante para que o jovem acabe por optar por outras áreas de conhecimento.

Nos últimos anos, a estratégia de aplicar a interdisciplinaridade, com o propósito de despertar vocações, por meio da interseção entre conteúdos de disciplinas do curso Técnico em Eletrotécnica, como Algoritmos e Programação e Circuitos Elétricos CC e CA, trouxeram resultados animadores.

Despertar o interesse dos alunos do ensino técnico em desenvolver programas de computador aliados à conteúdos de outras disciplinas da área, elevaram o nível dos trabalhos de Projetos Integradores desenvolvidos e apresentados ao final do curso, principalmente no que tange ao desenvolvimento de aplicativos.

Neste sentido, permitir que os alunos decidam quais passos tomar ao longo do desenvolvimento de um projeto são características importantes para que estes encontrem a melhor solução para os seus desafios.

\subsection{Fluxo geral de informações do programa}

A proposta para o desenvolvimento de um aplicativo para dispositivos móveis, que determinasse o valor da resistência de um resistor e a tolerância, surgiu como complementação de nota em uma das avaliações na disciplina de Circuitos Elétricos CC e CA. A proposta inicial era desenvolver o programa apenas utilizando a ferramenta VisuALG.

No bimestre em que os alunos estudaram os assuntos referentes a resistência elétrica e Leis de Ohm, as primeiras versões do programa em VisuALG foram desenvolvidas. Estruturas por eles aprendidas na disciplina de Algoritmos e Programação, como condicionais simples ou compostas, e estruturas de repetição, foram amplamente utilizadas.

Posteriormente, os discentes realizaram uma pesquisa sobre quais ferramentas de desenvolvimento de aplicativos poderiam ser utilizadas, e nesse sentido, por todos os pontos fortes citados anteriormente sobre a ferramenta, o Kodular foi escolhido. As etapas, definidas pelos alunos, que compreendem o fluxo geral de informações no programa (Figura 4) são:

1. O programa mostra em tela, para o usuário, a tabela de código de cores dos resistores;

2. O usuário informa se o resistor a ser determinado é de 4 ou 5 anéis;

3. Recebida essa informação do número de anéis, o programa solicita que o usuário informe a sequência de cores do resistor;

4. Os dois ou três primeiros dígitos referentes ao valor da resistência são determinados de acordo com o número associado a sequência de cores digitada;

5. O múltiplo é definido de acordo com o número associado a terceira ou quarta cor digitada;

6. Os valores referentes a faixas 1,2 ou 3 (5 anéis) são multiplicados pelo múltiplo correspondente;

7. O programa verifica qual o valor associado a cor do dígito 4 ou 5; dependendo do valor informado, o programa informa o valor de tolerância e o valor da resistência. 


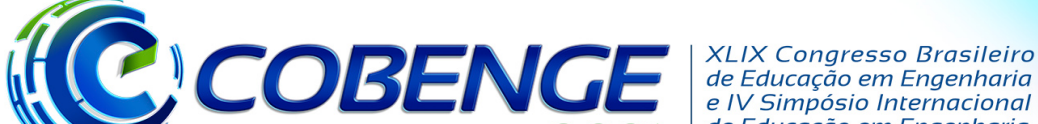 de Educação em Engenharia

Figura 4 - Fluxograma de funcionamento do aplicativo desenvolvido em Kodular, para resistores de 4 anéis.

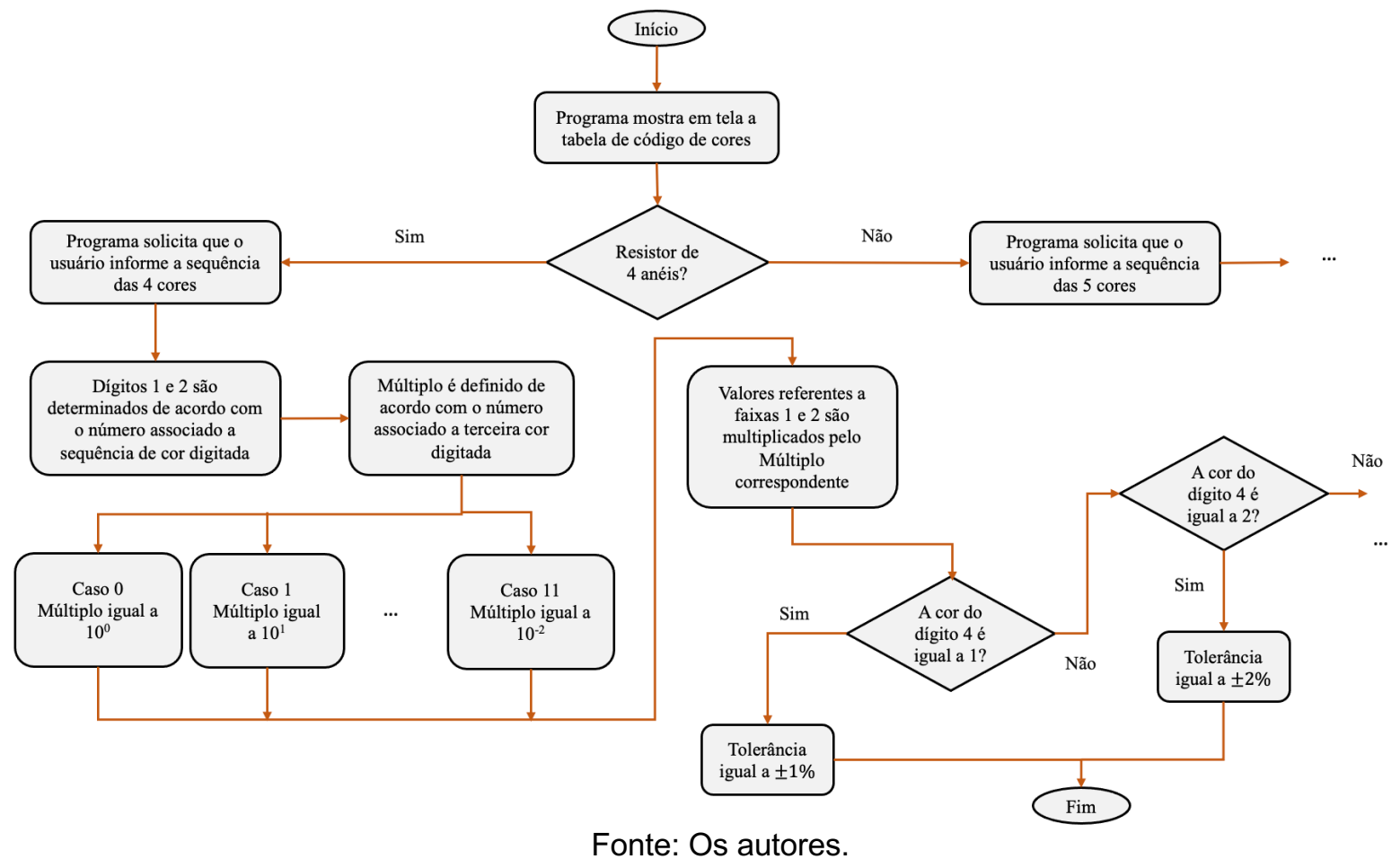

\section{RESULTADOS E CONCLUSÕES}

A estratégia de utilizar a interdisciplinaridade como ferramenta de motivação e despertar o interesse dos alunos nas diversas áreas de conhecimento não é uma novidade. Quando a construção do conhecimento é feita pelo aluno, a prática amplia o trabalho disciplinar na medida em que promove o desenvolvimento do saber como um todo. Nesse sentido, a sistemática de interação ensino/aprendizagem adotada, trouxe benefícios que vão além do atual contexto estudantil do aluno. A experiência adquirida pelos alunos durante o processo, despertou o interesse destes pelos cursos superiores de engenharia, além de agregar conhecimentos e experiências para posteriores trabalhos de iniciação científica e projetos de pesquisa dentro das universidades.

\section{REFERÊNCIAS}

\section{Livros:}

MARKUS, Otávio. Circuitos elétricos: corrente contínua e corrente alternada: teoria e exercícios. 9. Ed. Revisada, São Paulo: Editora Saraiva. 2011.

\section{Internet:}

MORA, N. D.; LUCAS, J. F. R.; MARAN, M. A. Materiais elétricos: compêndio de trabalhos. Disponível em: https://docplayer.com.br/1476904-Materiais-eletricoscompendio-de-trabalhos.html. Acesso em: 01 maio. 2021.

PUCRS. Código de cores para resistores. 2001. Disponível em:

https://www.inf.pucrs.br/ calazans/undergrad/laborg/cod_cores_res.html. Acesso em: 01 maio. 2021. 


\title{
INTERDISCIPLINARITY AS A STRATEGY TO STIMULATE DEVELOPMENT OF COMPUTATIONAL TOOL TO TEACHING SUPPORT IN THE AREA OF ELECTRICAL
}

\begin{abstract}
This work presents the result of the interdisciplinarity used as a strategy for stimulus the development of a computational tool to teaching support in the field of electrotechnics. The focus of the work is found in the set of actions carried out by the students, from the Technical course in Electrotechnics at IFPA, during the planning and execution of the steps that culminated in the development of an application for mobile device. The application, developed using a Kodular tool, automated the process of determining the resistance and tolerance value for resistors of 4 and 5 electronic color code.
\end{abstract}

electronic color code

Keywords: Interdisciplinarity. Application development. Electric circuits. Electrical. 